\title{
Protective Effect of Shenqi Maixintong Capsule against ox-LDL-Induced Injury in RAW264.7 Macrophages
}

\author{
Qianqian Ma $\left(\mathbb{D},{ }^{1}\right.$ Min Liang $\mathbb{D},{ }^{2}$ Tiange Cai $\left(\mathbb{D},{ }^{3}\right.$ Yingjia $Y u^{2}{ }^{2}$ Xinjian Liang, ${ }^{2}$ \\ Qingzhuang Chen, ${ }^{2}$ Manling Du, ${ }^{2}$ Yong Ouyang $\mathbb{D}^{2},{ }^{2}$ Chaojun Chen $\mathbb{D}^{2},{ }^{2}$ and Yu Cai $\mathbb{D}^{1,4,5}$ \\ ${ }^{1}$ College of Pharmacy, Jinan University, Guangzhou 510632, China \\ ${ }^{2}$ Guangzhou Hospital of Integrated Traditional Chinese and Western Medicine, Guangzhou 510800, China \\ ${ }^{3}$ College of Life Sciences, Liaoning University, Shenyang 110036, China \\ ${ }^{4}$ Cancer Research Institute of Jinan University, Guangzhou 510632, China \\ ${ }^{5}$ International Cooperative Laboratory of Traditional Chinese Medicine Modernization and Innovative Drug Development of \\ Chinese Ministry of Education (MOE), School of Pharmacy, Jinan University, Guangzhou 510632, China
}

Correspondence should be addressed to Yong Ouyang; oyylm@126.com, Chaojun Chen; ccjbs@126.com, and

YuCai; caiyu8@sohu.com

Received 16 December 2019; Revised 2 March 2020; Accepted 10 March 2020; Published 21 April 2020

Academic Editor: Antonella Di Sotto

Copyright ( $) 2020$ Qianqian Ma et al. This is an open access article distributed under the Creative Commons Attribution License, which permits unrestricted use, distribution, and reproduction in any medium, provided the original work is properly cited.

\begin{abstract}
Shenqi Maixintong capsule (SMC), a traditional Chinese medicine compound, exerts various therapeutic effects, including nourishing one's vitality, improving blood circulation, regulating vital energy, and promoting coronary circulation. Studies have demonstrated that SMC could effectively alleviate atherosclerosis (AS) and delay its development. However, the effect of SMC on lipid metabolism remains unclear. Herein, we investigated the mechanism of SMC on lipid metabolism of RAW264.7 cells induced by oxidized low-density lipoprotein (ox-LDL). Drug-containing serum was prepared by intragastric administration of SD (Sprague Dawley) rats. RAW264.7 cells were transformed into foam cells with $25 \mathrm{mg} / \mathrm{L}$ ox-LDL. SMC-treated rat serum increased the survival rate of ox-LDL-induced RAW264.7 cells. Oil Red staining, total cholesterol, and free cholesterol detection data showed that the intermediated dose of SMC had the best effect on reducing the lipid accumulation, lipid droplets, intracellular cholesterol, and total cholesterol content of RAW264.7 cells. The results of western blotting showed that the expression of ABCA1, ABCG1, LXR $\alpha, \operatorname{PPAR} \gamma$, and peroxisome proliferation-activated receptor alpha (PPAR $\alpha$ ) was increased by SMC, and proprotein convertase subtilisin/kexin type 9 (PCSK9) was reduced, which promoted reverse cholesterol transport (RCT) of RAW264.7 cells and inhibited foam cell formation. Furthermore, SOD and MDA data indicated that SMC could reduce the senescence of RAW264.7 cells. These findings suggest that SMC might prevent ox-LDL-induced damage of macrophages in AS patients by improving cell viability and slowing down lipid accumulation and senescence.
\end{abstract}

\section{Introduction}

Atherosclerosis (AS) is a common disease and the major cause of morbidities and mortalities worldwide. This is a complex pathological process and is an abnormal regulation involving multiple factors. It is widely accepted that the aggregation and foaming of cholesterol and cholesterol esters in macrophages play an important role in the formation of AS [1-3], and elevated circulating low-density lipoprotein cholesterol (LDL-c) represents the major independent risk factor for AS [4]. Therefore, lowering LDL levels and blocking foam cell formation may be the key to reducing AS [5].

Numerous studies have shown that the classical PPAR $\alpha$ LXR $\alpha$-ABCA1, PPAR $\gamma$-LXR $\alpha$-ABCA 1 , and LXR $\alpha$-ABCA 1 signaling pathways involved in nuclear receptors mediate oxidized lipids in foam cells [6-10]. The proteins involved in these signaling pathways transfer excess lipids in the cell to the high-density lipoprotein (HDL) in the periphery for reverse transport to intrahepatic metabolism [11]. ABCG1 
has a critical role in mediating cholesterol efflux to HDL and preventing cellular lipid accumulation [12-14]. ABCA1 and ABCG1 can be highly induced in macrophages [15]. It was found that the expression of ABCA1 is mainly regulated by nuclear receptors $\operatorname{LXR} \alpha$ and $\operatorname{LXR} \beta$, while $\operatorname{LXR} \alpha$ is regulated by PPAR $\gamma[16,17]$. Then, PPAR $\gamma$ and $\operatorname{LXR} \alpha$ can affect cholesterol transport by inducing the expression of ABCA1 [18-20]. Previous studies have shown that PCSK9 increases expression in macrophages, causing inflammation and cholesterol accumulation by inhibiting RCT action [21, 22].

SMC is a Chinese herbal compound first developed by Guangzhou hospital of integrated traditional Chinese and Western medicine. It is prepared from the eight herbs of Radix Ginseng Rubra, Radix Notoginseng, Rhizoma Ligustici Chuanxiong, Radix Salviae Miltiorrhizae, Radix Astragali seu Hedysaari, Rhizoma Cyperi, Fructus Crataegi, and Fructus Lycii. with modern Chinese medicine preparation technology. It has been used in clinical application for more than 20 years and has a definite effect on atherosclerosis [23].

40 patients with cervical atherosclerotic plaque were treated with SMC and observed changes in plaque after 2 courses of treatment. The total effective rate was 72.5\% [24]. Another clinical trial of 70 patients showed that SMC can effectively lower blood lipid levels and alleviate the disease [25]. Other studies have also shown that SMC has the effect of lowering blood lipids, improving vascular endothelial function, and delaying atherosclerosis [26-29].

Although SMC has an active role in stabilizing atherosclerotic lipid plaques, its mechanism is not clear. It is necessary to further study the mechanism of SMC regulating lipid metabolism. Therefore, ox-LDL was used to induce the RAW264.7 macrophage formation cell injury model, and the effect of SMC on cell lipid metabolism was evaluated.

\section{Materials and Methods}

2.1. Materials. SMC was purchased from Guangzhou hospital of integrated traditional Chinese and Western medicine, Guangzhou, China (batch numbers: 180501, 180601 and 180701; approval number: ZB20120001). Ginsenoside Rg1 (99.5\% purity) and ferulic acid (98.9\% purity) were purchased from National Institutes for Food and Drug Control, China (batch numbers: 110703-201730 and 110773201012). Murine macrophage RAW264.7 cells and CCK8 kit were purchased from NanJing KeyGen Biotech Co., Ltd., China. Dulbecco's modified eagle medium (DMEM) and RPMI-1640 were purchased from Gibco (Thermo Fisher Scientific, Inc., USA), FBS was purchased from AusGeneX pty Ltd., Australia. Oxidized low-density lipoprotein (oxLDL) was purchased from Shanghai Yuanye Bio-Technology Co., Ltd. Pentobarbital Sodium was purchased from Shanghai Univ Biotech Co., Ltd., China. Anti-ABCA1, antiABCG1, anti-PPAR $\alpha$, anti-PPAR $\gamma$, anti-PCSK9, and all these rabbit anti-mouse monoclonal antibodies were purchased from Cell Signaling Technology, Inc., USA. AntiLXR $\alpha$, HRP-labeled goat, and anti-rabbit G secondary antibody were obtained from Abcam, USA. Oil Red O staining kit, SOD, and MDA kit were purchased from Nanjing Jiancheng Bioengineering Institute, China.
2.2. Animals. 40 male SD (Sprague Dawley) rats (180-220 g, 4-5 weeks old) were provided by Jinan Pengyue Experimental Animal Breeding Co., Ltd., license number SCXK (Lu) 20140007. All SD rats were housed under standard conditions (room temperature $23 \pm 2^{\circ} \mathrm{C}$, humidity $60 \pm 15 \%$, $12 \mathrm{~h} / 12 \mathrm{~h}$ light/dark cycles) and given free access to standard rodent chow and water. All experimental procedures were performed in accordance with the Guidelines for Animal Experiments from the Committee of Medical Ethics, National Health Department of China.

\subsection{Drug Content Determination. Radix ginseng rubra,} Panax notoginseng, and Ligusticum chuanxiong in the Chinese herbal compound improve blood circulation and remove blood stasis, and these three are the major ingredients in the SMC compound. Therefore, the main active ingredients of these traditional Chinese herbs, ginsenoside Rg1 and ferulic acid, were selected as the calibration materials, and the drug content was determined according to the Chinese Pharmacopoeia 2015 edition. To control the SMC quality, the high-performance liquid chromatography (HPLC) method was performed to establish the fingerprint spectrum. The analyses were performed with an Agilent 1260 system (Agilent Technologies, Hewlett-Packard-strasse-8, 76337 Waldbronn, Germany) equipped with a quaternary gradient pump. The components were eluted with a system consisting of water (A) and acetonitrile (B), and the elution was $A(75 \%)$ and $B(25 \%)$. The chromatographic column was Agilent ZORBA $\times$ SB-C18 $(4.6 \times 250 \mathrm{~mm}, 5 \mu \mathrm{m})$. The mobile phase flow rate was $0.8 \mathrm{~mL} / \mathrm{min}$, and the column temperature was maintained at $25 \pm 1^{\circ} \mathrm{C}$. The detection wavelength was $203 \mathrm{~nm}$ (ginsenoside Rg1) and $316 \mathrm{~nm}$ (ferulic acid) with an injection volume of $10 \mu \mathrm{L}$. Ginsenoside Rg1 and ferulic acid were dissolved in methanol as control and measured by the HPLC. The specificity, linearity, precision, repeatability, stability, and recovery were further tested.

\subsubsection{SMC Sample Preparation with Ginsenoside Rg1.} Take $5 \mathrm{~g}$ of SMC powder, put it in a Soxhlet extractor, add chloroform, and heat to reflux for $3 \mathrm{~h}$. Take out the medicine residue, put it into the Erlenmeyer flask, add $50 \mathrm{~mL}$ of watersaturated n-butanol, and let it stand overnight. Sonicate for $30 \mathrm{~min}$, filter and accurately measure $25 \mathrm{~mL}$, and evaporate the solution to dryness. The residue was dissolved in methanol, diluted to $5 \mathrm{~mL}$, and filtered.

2.3.2. SMC Sample Preparation with Ferulic Acid. Take $1 \mathrm{~g}$ of SMC powder and place in an Erlenmeyer flask, and then $10 \mathrm{~mL}$ of $70 \%$ methanol was added and sealed. Weigh and heat reflux for $30 \mathrm{~min}$. After cooling, weigh again and make up the lost weight with $70 \%$ methanol. Mix and filter the supernatant.

2.4. Preparation of Drug-Containing Serum. 40 SPF (specificpathogen-free) healthy SD rats, male, weighing 180-200 g, were used in this study. After 10 days of adaptive feeding, they were randomly divided into blank group, low-dose 
group, middle-dose group, high-dose group, and 10 per group. SMC adult dosage was 4 capsules/time, 3 times/day, i.e., a total of 12 capsules daily. The rat dose was calculated according to "equivalent dose ratio between human and animal converted by body surface area" [30]. After soaking for $1 \mathrm{~h}$, the SMC were prepared by water extraction twice. The extract was filtered and condensed to make the concentration equivalent to 2.0 capsules per $\mathrm{mL}$ and then kept at $4^{\circ} \mathrm{C}$. Also, the final concentration was 0.27 capsules per $\mathrm{mL}$ (low), 0.54 capsules per $\mathrm{mL}$ (middle), and 1.08 capsules per $\mathrm{mL}$ (high) and was administered at a dose of $1 \mathrm{~mL} / 100 \mathrm{~g}$. Every test group was administered SMC at $1 \mathrm{~mL} / 100 \mathrm{~g} / \mathrm{d}$, and the blank control group was given normal saline once a day $1 \mathrm{ml} / 100 \mathrm{~g}$. The rats were intragastrically administered for 8 days, fasted for $12 \mathrm{~h}$ before the last administration, and anesthetized with $0.3 \%$ sodium pentobarbital $1 \mathrm{~h}$ after the administration. Blood was taken from the femoral artery, and serum was separated after centrifugation and filtered for use.

2.5. RAW264.7 Macrophage Cell Culture. RAW264.7 cells were cultured in a DMEM medium containing $10 \%$ FBS in a humidified atmosphere at $37^{\circ} \mathrm{C}, 5 \% \mathrm{CO}_{2}$. Then, the cells were passaged three times. In the logarithmic growth phase, the cells were randomly divided into five groups: control group, ox-LDL group, low-dose group, intermediated-dose group, and high-dose group, at least three replicates for each group, and cell counting was performed so that the number of cells in each group was the same. The cells were cocultured with $25 \mathrm{mg} / \mathrm{L}$ ox-LDL for $48 \mathrm{~h}$, except the blank group, and then replaced with normal medium (vehicle medium), blank medium, low-dose medium, intermediated-dose medium, and high-dose drug-containing medium for $24 \mathrm{~h}$. Subsequently, the cells were collected and extracted for analysis.

2.6. Cell Proliferation Assay. RAW264.7 cells were seeded into 96 -well plates $\left(10^{3} /\right.$ well $)$, and $100 \mu \mathrm{L}$ medium was added into each well. 96-well plates were cultured for $24 \mathrm{~h}$ in a cell culture incubator containing $5 \% \mathrm{CO}_{2}$ at $37^{\circ} \mathrm{C}$. Then, the cells in the 96-well plate are administered to different concentrations of ox-LDL $(0,20,40,60$, or $100 \mathrm{mg} / \mathrm{L})$, different concentrations of drug-containing serum, blank (vehicle medium), and a mixture of ox-LDL + drug-containing serum. After $24 \mathrm{~h}$ of culture, cell proliferation assays were performed using a CCK8 kit and a microplate reader $(490 \mathrm{~nm})$.

2.7. Oil Red O Staining. The well-grown macrophages were seeded in a 6-well plate at a density of $1 \times 10^{5} /$ well, cultured for $24 \mathrm{~h}$, and the cell culture medium was discarded after the cells were attached. ox-LDL was prepared in a RPMI-1640 medium, and the final concentration was $20-30 \mathrm{mg} / \mathrm{L}$. The prepared ox-LDL was added to a 6 -well plate for $48 \mathrm{~h}$ to establish a foam cell model. Identification of foam cells by Oil Red O staining was carried out. After gently washing the cells 3 times with PBS, 4\% paraformaldehyde was fixed at room temperature for $30 \mathrm{~min}$. After washing, the cells were again incubated at room temperature with Oil Red $\mathrm{O}$ solution for $30 \mathrm{~min}$. Discard the staining solution, fix it with $60 \%$ isopropyl alcohol for $5 \sim 10 \mathrm{~s}$, and rinse immediately with PBS 2-3 times. The morphology of the foam cells was observed under a microscope, and it was found that the intracellular bright red lipid droplets were foam cells. Oil Red $\mathrm{O}$ imaging was taken using the $\times 40$ objective lens. Also, quantitative analysis of intracellular lipid droplets was performed using integrated optical density (IOD) and Image J software (National Institutes of Health, Bethesda, MD, USA).

2.8. Total Cholesterol and Free Cholesterol Assays. RAW264.7 cells were pretreated and cultured according to the abovementioned method. Then, the kit instructions were followed. After treatment, the cells were washed once with PBS, and the cells were lysed by ultrasonic homogenization, heated at $70^{\circ} \mathrm{C}$ for $10 \mathrm{~min}$, and centrifuged at $2000 \mathrm{rpm}$ for $5 \mathrm{~min}$ at room temperature. The supernatant was removed, and total cholesterol and free cholesterol were measured using a microplate reader $(550 \mathrm{~nm})$. Protein quantification was performed using the BCA method. And samples of total cholesterol and free cholesterol were tested for protein quantification.

2.9. SOD Measurement. The cells were cultured as described above, and each group of cells was collected and operated according to the SOD detection kit. The original cell culture medium was discarded, and PBS was washed 2-3 times, and then the cells were collected, centrifuged at $1500 \mathrm{rpm}$ for $10 \mathrm{~min}$, and precipitated. Buffer was added, and the cells were sonicated. After that, the reagent was added to the cells according to the kit instruction. Then, the cells were incubated at $37^{\circ} \mathrm{C}$ for $20 \mathrm{~min}$ and the SOD activity was read at $450 \mathrm{~nm}$.

2.10. MDA Measurement. The pretreatment of the cells is the same as the treatment for the SOD measurement. Heating was then carried out at $95^{\circ} \mathrm{C}$ for $20 \mathrm{~min}$. The reagents required for the experiment were prepared and tested according to the MDA kit instructions. The absorbance was read at $530 \mathrm{~nm}$.

2.11. Western Blot Assay. RAW264.7 cells were treated as described and washed three times with PBS, and cell lysates were generated using RIPA lysis buffer with PMSF. Blow off the cells and transfer the solution into a new EP tube. The solution was lysed on ice for $30 \mathrm{~min}$ and centrifuged at $12,000 \mathrm{rpm}$ for $30 \mathrm{~min}$ at $4^{\circ} \mathrm{C}$. Then, the supernatant was assayed for protein using a BCA kit. The protein concentration was adjusted with RIPA lysate, and 5x loading buffer was added and boiled for $5 \mathrm{~min}$. Then, the proteins were stored at $-80^{\circ} \mathrm{C}$. Protein lysate was loaded onto $10 \%$ SDSPAGE gel, electrophoresed, and then transferred to the polyvinylidene fluoride membrane. The membranes were probed with antibodies against ABCA1, ABCG1, $\mathrm{LXR} \alpha$, $\operatorname{PPAR} \alpha, \operatorname{PPAR} \gamma$, and PCSK9, respectively. Signals were 
amplified and observed with the horseradish peroxidase (HRP)-conjugated secondary antibody (Cell Signaling, CA, U.S.A., 1:5000 dilution) and enhanced chemiluminescence. Densitometry was detected with an ECL Western Blot Detection System (4A Biotech, Beijing, China). All experiments were repeated at least three times.

2.12. Statistical Analyses. Statistical analysis was performed using SPSS 22.0 software. Data are presented as mean\pm standard deviation. Statistical analysis was performed using one-way ANOVA, and post-hoc comparisons were carried out using Duncan's multiple-range test. $P<0.05$ was considered statistically significant.

\section{Results}

3.1. Determination of Drug Content. To determine the quality of the SMC, two major compounds were used as quality control standards for SMC. The HPLC chromatogram of SMC was achieved on a C18 column using a mixture of acetonitrile and water with $0.1 \%$ phosphoric acid as the mobile phase. The HPLC chemical fingerprint of SMC is shown in Figure 1.

The average ginsenoside $\mathrm{Rg} 1$ content of the three batches of SMC samples was $14.05 \mathrm{mg} / \mathrm{g}$. The linear regression equation and correlation coefficient of ginsenoside Rg1 were calculated as follows: $y=514.53 x+355.21, R 2=0.9996$, and $r=0.9997$, indicating that the linear relationship of ginsenoside Rg1 is good in the range of 5.39 50.30 $\mu$ g. The RSD (relative standard deviation) values of the precision, repeatability, and stability test results are less than $2 \%$, and the RSD value of the recovery test is $2.53 \%$, indicating that the experimental research method is accurate and reliable, and the recovery rate experiment results are shown in Table 1.

The average ferulic acid content of the three batches of SMC samples was $2.42 \mathrm{mg} / \mathrm{g}$. The linear regression equation and correlation coefficient of ferulic acid are as follows: $y=4153.3 x+37.111, R^{2}=0.999$, and $r=0.9995$, indicating that the linear relationship of ferulic acid is good in the range of $0.046 \sim 0.396 \mu \mathrm{g}$. Similarly, the RSD values of the methodological tests are less than $2 \%$, and the recovery rate experiment results are shown in Table 2.

3.2. Improvement of ox-LDL-Induced RAW264.7 Cell Viability by SMC. The cell viability of RAW264.7 cells was evaluated, and Figure 2(a) shows that ox-LDL reduced the cell viability of RAW264.7 cells in a dose-dependent manner. Figure 2(b) shows that half of the cells survived at $100 \mathrm{mg} / \mathrm{L}$ ox-LDL and SMC was not cytotoxic to RAW264.7 cells at intermediated dose. In Figure 2(c), intermediate-dose SMC treatment of ox-LDL-induced RAW264.7 cells significantly increased cell viability. Therefore, the Chinese herbal compound SMC can rescue ox-LDL-induced RAW264.7 cells from death.

3.3. Inhibition of Lipid Accumulation by SMC in ox-LDLInduced RAW264.7 Cells. Next, we measured the cellular lipid level of RAW264.7 cells after $24 \mathrm{~h}$ ox-LDL treatment. The Oil Red O staining showed that the lipid droplets in the ox-LDL + SMC groups were significantly less than those in the ox-LDL group (Figure 3). There was significant difference between control and ox-LDL groups $(P<0.001)$, and the $p$ value of the ox-LDL + intermediated group was less significant than that of the ox-LDL group $(P<0.001)$.

The total cholesterol and free cholesterol levels showed that low and intermediated dose of SMC significantly reduced total cholesterol $(P<0.01)$ and free cholesterol $(P<0.01)$ induced by ox-LDL (Figure 4$)$. Therefore, SMC could inhibit ox-LDL-induced lipid accumulation in RAW264.7 cells.

3.4. Effects of SMC on SOD Activity and MDA in RAW264.7 Cells. RAW264.7 cells were cultured separately by SMC and ox-LDL treated together and ox-LDL alone, and then the SOD and MDA contents were determined. The data in Figure 5(a) show that the SOD activity of ox-LDL was lower than that of the other groups and the difference was statistically significant $(P<0.01)$. Different dose groups of SMC increased the decrease in SOD value caused by ox-LDL to varying degrees, and the groups of SMC alone did not significantly reduce the SOD value. MDA in the ox-LDL group was higher than the other groups, which was statistically significant $(P<0.01)$ (Figure $5(b))$. Different dose groups of SMC reduced the increase in MDA values caused by ox-LDL to varying degrees, and every dose group did not significantly change the MDA value of each group. Therefore, SMC increased the SOD activity after ox-LDL treatment and decreased the content of MDA.

3.5. Regulation of the Expression of ABCA1, ABCG1, $L X R \alpha$, PPAR $\alpha$, PPAR $\gamma$, and PCSK9 in RAW264.7 Cells. It has been shown that ABCA1, ABCG1, and other related proteins are related to the regulatory mechanism of cellular cholesterol transport. We were wondering whether SMC indeed rescues the macrophage survival by activating this blah, the blah pathway. To test this hypothesis, we treated the RAW cells in vitro with SMC and measured ABCA1, ABCG1, LXR $\alpha$, $\operatorname{PPAR} \alpha, \operatorname{PPAR} \gamma$, and PCSK9 by western blotting. Our data show (Figure 6) that ox-LDL decreased the expression of ABCA1, ABCG1, LXR $\alpha$, PPAR $\alpha$, and PPAR $\gamma$ but increased PCSK9 expression. After treatment with SMC, ABCA1, ABCG1, LXR $\alpha, \operatorname{PPAR} \alpha$, and $\operatorname{PPAR} \gamma$ expressions were upregulated and the expression of PCSK9 was downregulated. Thus, SMC can modify the expression of ABCA1, ABCG1, LXR $\alpha$, PPAR $\alpha$, PPAR $\gamma$, and PCSK9 in RAW264.7 cells.

\section{Discussion and Conclusions}

Foam cell formation is the key step in the development of AS. More and more studies have shown that macrophage uptake of ox-LDL by scavenger receptors (SRs) leads to significant accumulation of cholesterol, transforming macrophages into foam cells and promoting the development of AS [31-33]. Here, we used an ox-LDL-induced 

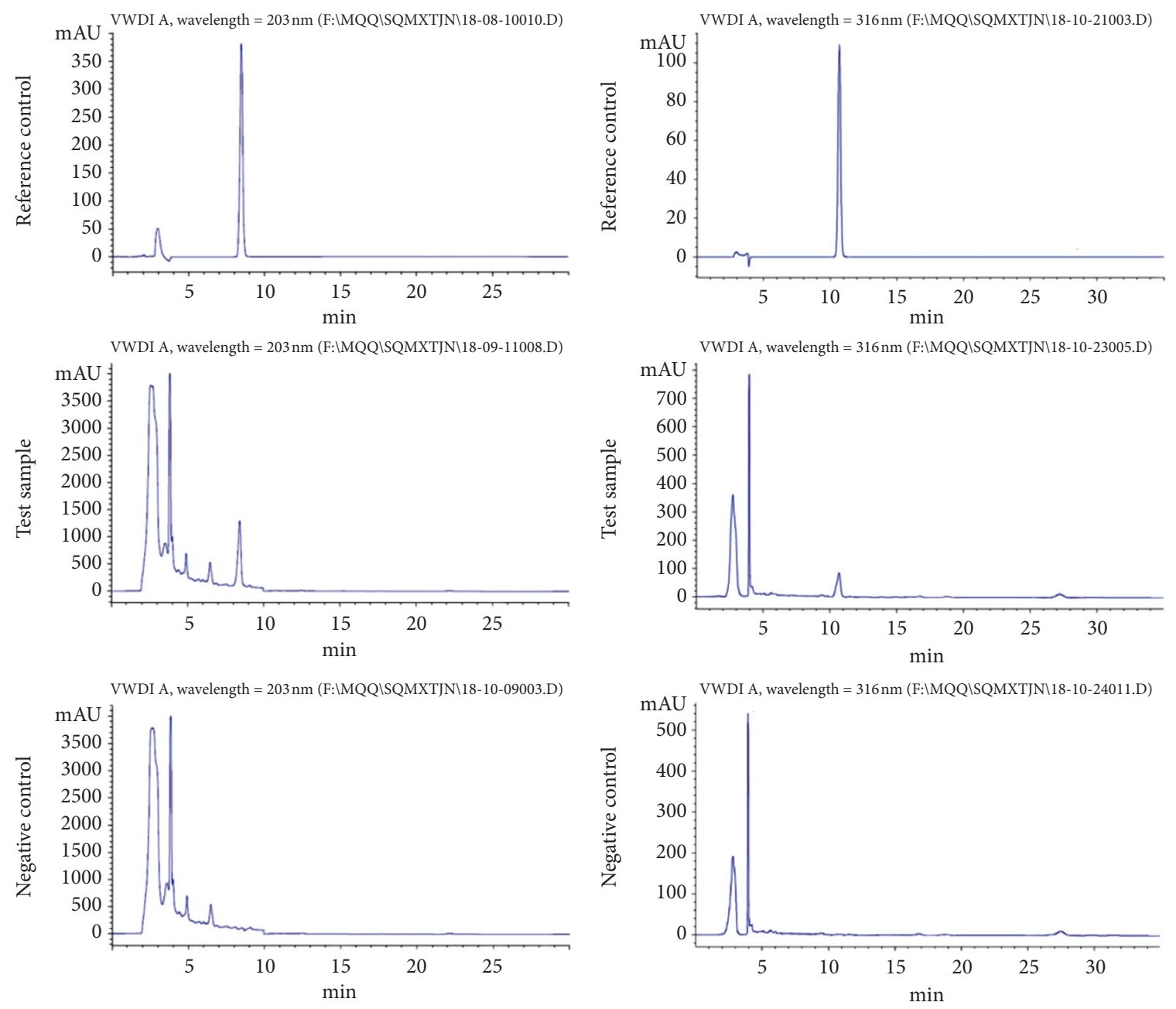

(a)

(b)

FiguRE 1: HPLC of ginsenoside Rg1 and ferulic acid. (a) Chromatogram of ginsenoside Rg1. (b) Chromatogram of ferulic acid. The reference substance solution and the test solution showed chromatographic peaks at the same time (ginsenoside Rg1: 8.43 min; ferulic acid: $10.68 \mathrm{~min}$ ), and the negative control solution did not show the corresponding peak.

TABle 1: Ginsenoside Rg1 recovery rate test.

\begin{tabular}{|c|c|c|c|c|c|c|c|}
\hline No. & Sample volume (g) & Sample content (mg) & Addition (mg) & Measured (mg) & Recovery (\%)-> & Average recovery rate (\%) & RSD (\%) \\
\hline 1 & 0.5096 & 7.05 & 3.51 & 10.46 & 97.15 & \multirow{6}{*}{99.44} & \multirow{6}{*}{2.53} \\
\hline 2 & 0.5065 & 7.01 & 3.51 & 10.64 & 103.41 & & \\
\hline 3 & 0.5021 & 6.95 & 7.02 & 13.88 & 98.72 & & \\
\hline 4 & 0.4972 & 6.88 & 7.02 & 13.70 & 97.15 & & \\
\hline 5 & 0.5047 & 6.98 & 10.53 & 17.37 & 98.67 & & \\
\hline 6 & 0.4986 & 6.90 & 10.53 & 17.59 & 101.52 & & \\
\hline
\end{tabular}

TABLE 2: Ferulic acid recovery rate test.

\begin{tabular}{|c|c|c|c|c|c|c|c|}
\hline No. & Sample volume (g) & Sample content (mg) & Addition (mg) & Measured (mg) & Recovery (\%)-> & Average recovery rate $(\%)$ & RSD (\%) \\
\hline 1 & 0.5084 & 1.209 & 0.602 & 1.808 & 99.50 & \multirow{6}{*}{99.21} & \multirow{6}{*}{0.84} \\
\hline 2 & 0.5062 & 1.205 & 0.602 & 1.796 & 98.17 & & \\
\hline 3 & 0.5024 & 1.196 & 1.193 & 2.395 & 100.50 & & \\
\hline 4 & 0.4989 & 1.187 & 1.193 & 2.361 & 98.41 & & \\
\hline 5 & 0.5018 & 1.194 & 1.797 & 2.978 & 99.28 & & \\
\hline 6 & 0.4994 & 1.189 & 1.797 & 2.975 & 99.39 & & \\
\hline
\end{tabular}




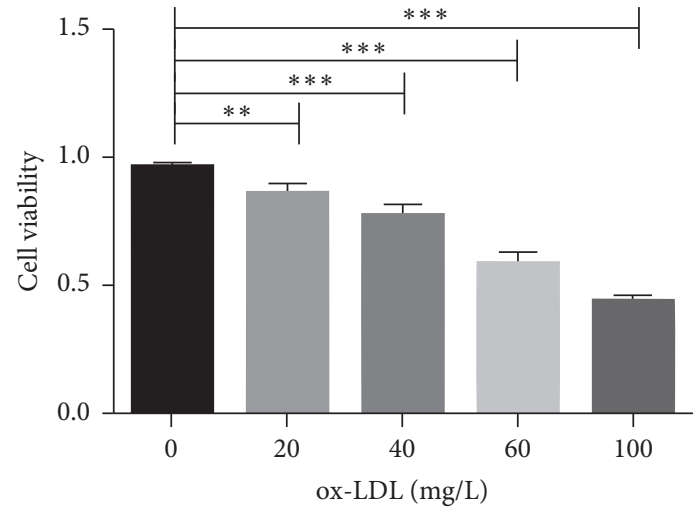

(a)

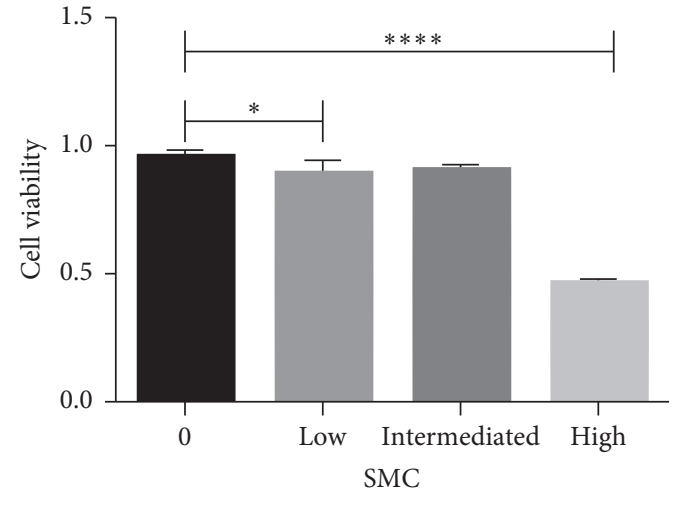

(b)

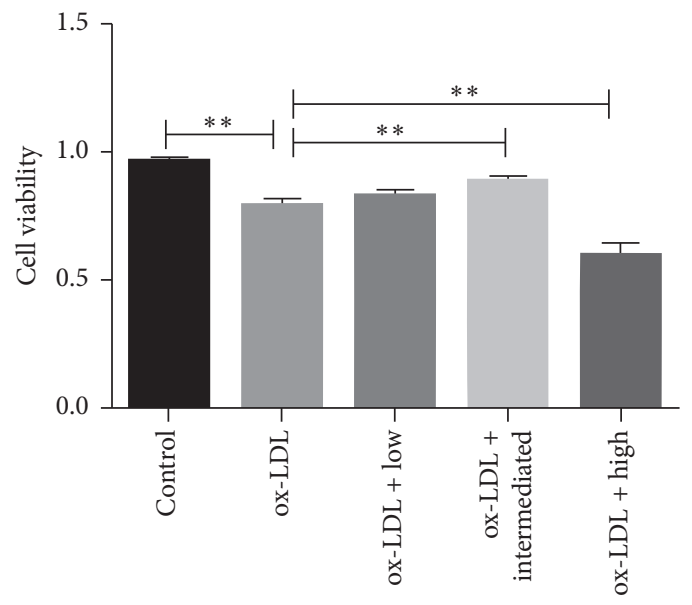

ox-LDL + SMC

(c)

FigURE 2: SMC and RAW264.7 macrophage cell viability. RAW264.7 cells were treated with (a) ox-LDL, (b) SMC of low, intermediated, and high dose, or (c) SMC of low, intermediated, and high dose + ox-LDL, and the cell viability was determined. ox-LDL reduced RAW264.7 cell viability in a concentration-dependent manner, and half of the cells were viable with $100 \mathrm{mg} / \mathrm{L}$ ox-LDL (Figure 2(a). SMC was not cytotoxic to RAW264.7 cells at intermediated dose (Figure 2(b). Treatment of ox-LDL-induced RAW264.7 cells with intermediated-dose SMC significantly improved viability (Figure 2 (c). Data are presented as mean \pm standard deviation. ${ }^{* *} P<0.01$; ${ }^{* * *} P<0.001$. ox-LDL, oxidized low-density lipoprotein; SMC, Shenqi Maixintong capsule.

Control

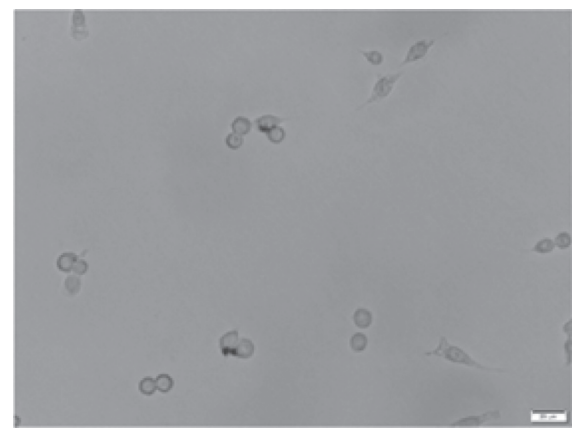

(a)

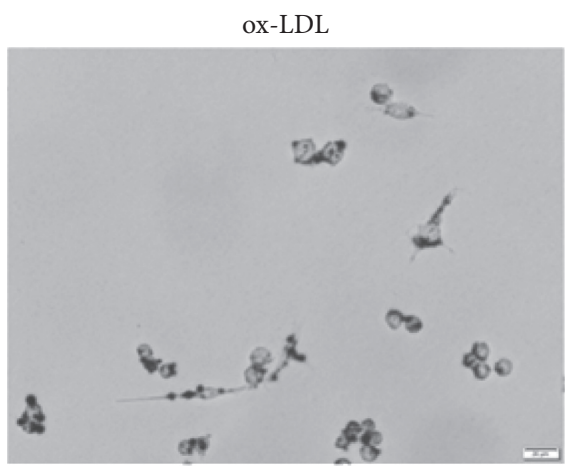

(b)

Figure 3: Continued. 


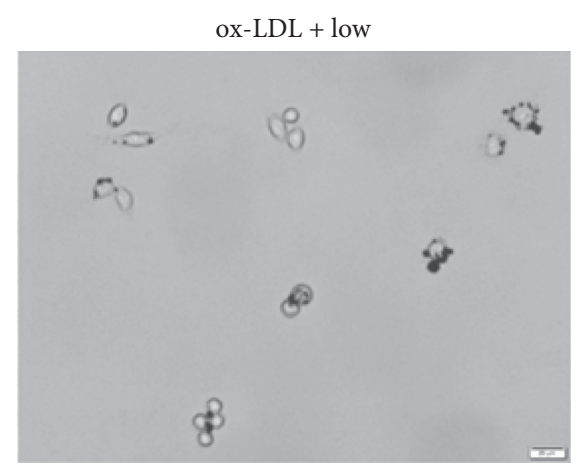

(c)

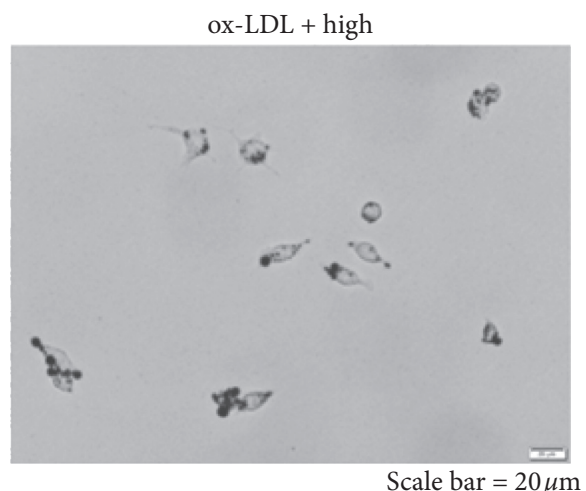

(e)

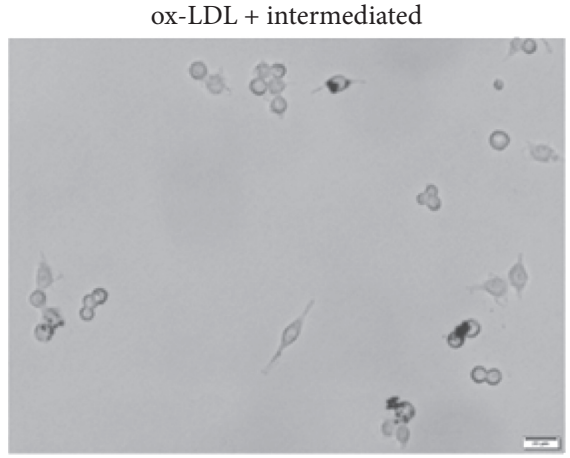

(d)

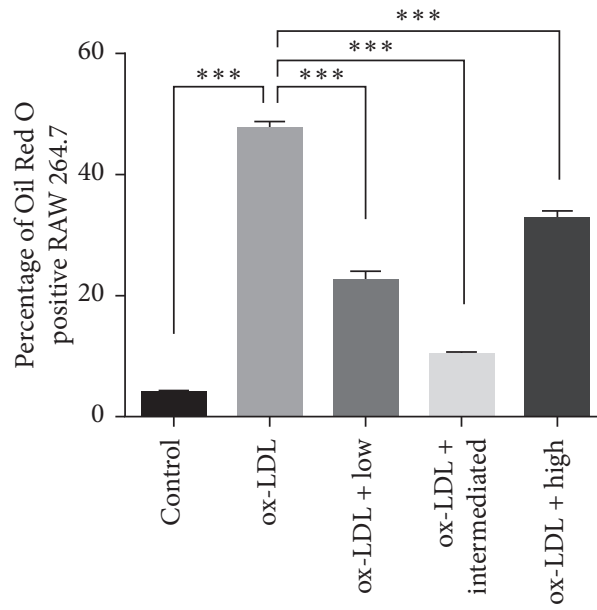

(f)

FIGURE 3: SMC of low, intermediated, and high dose and lipid accumulation in RAW264.7 cells induced by ox-LDL. Oil Red O staining of RAW264.7 cells was carried out, RAW264.7 cells were treated with SMC and ox-LDL, and the lipid accumulation was measured by Oil Red $\mathrm{O}$ staining. The bar graph shows the percentage of Oil Red $\mathrm{O}$ staining in different groups. There was significant difference between control and ox-LDL groups $(P<0.001)$. Lipid droplets in the ox-LDL + SMC groups were significantly less than those in the ox-LDL group, and the $p$ value of the ox-LDL + intermediated group was less significant than that of the ox-LDL group $(P<0.001)$. Original magnification: 400x. Data are presented as mean \pm standard deviation. ${ }^{*} P<0.05,{ }^{* *} P<0.01$, and ${ }^{* * *} P<0.001$. ox-LDL, oxidized low-density lipoprotein; SMC, Shenqi Maixintong capsule; low, low dose of SMC; intermediated, intermediated dose of SMC; high, high dose of SMC.

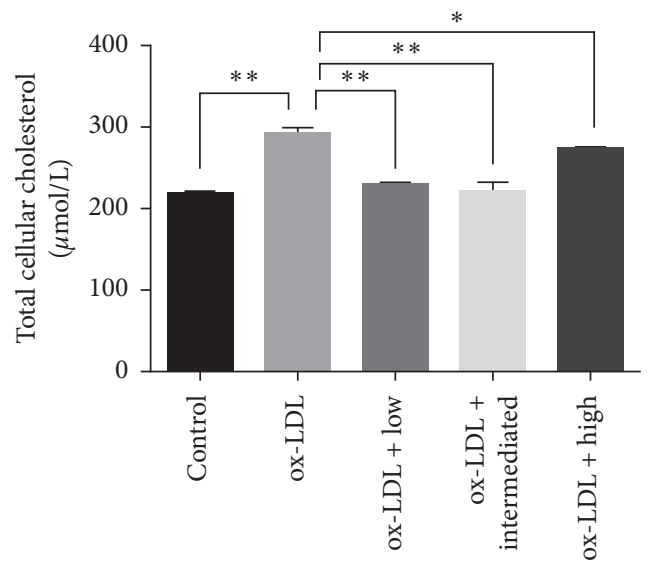

(a)

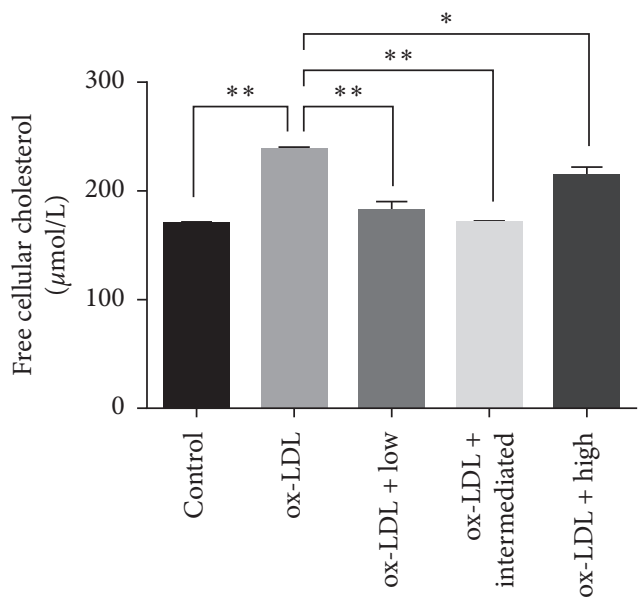

(b)

FIGURE 4: Determination of total cholesterol and free cholesterol. (a) RAW264.7 cells were treated with SMC of low, intermediated, and high dose and ox-LDL, and the total cellular cholesterol was measured. (b) RAW264.7 cells were treated with SMC of low, intermediated, and high dose and ox-LDL, and the free cellular cholesterol was measured. Low and intermediated dose of SMC significantly reduced total cholesterol and free cholesterol induced by ox-LDL $(P<0.01)$. Data are presented as mean \pm standard deviation. ${ }^{*} P<0.05 ;{ }^{* *} P<0.01$. oxLDL, oxidized low-density lipoprotein; SMC, Shenqi Maixintong capsule; low, low dose of SMC; intermediated, intermediated dose of SMC; high, high dose of SMC. 


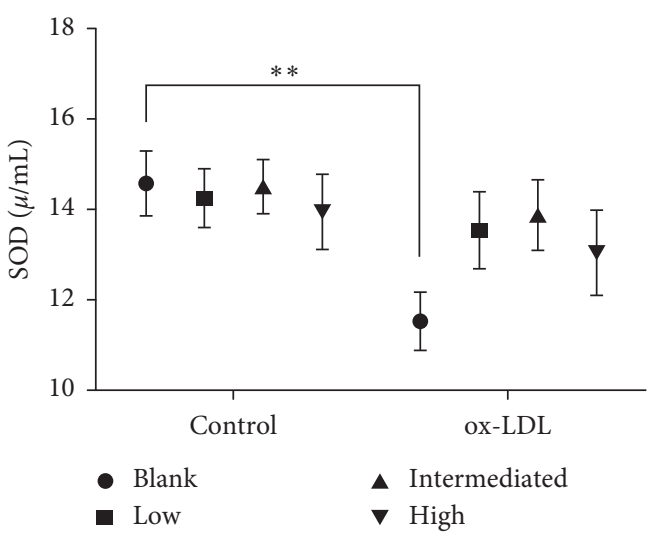

(a)

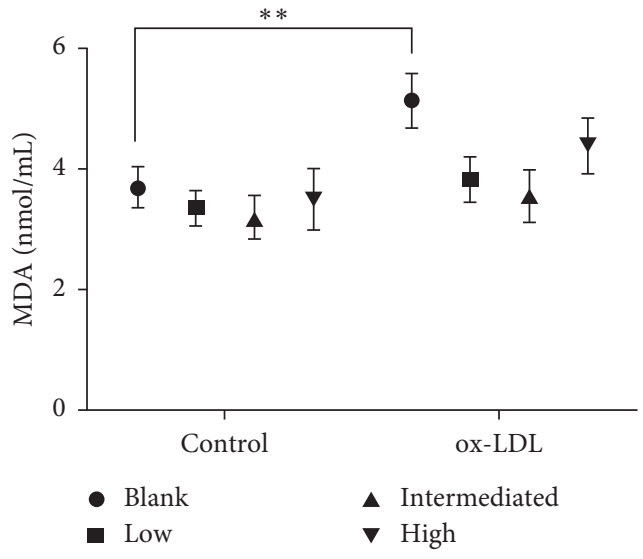

(b)

FIGURE 5: Cell SOD activity and MDA with ox-LDL. (a) SOD activity measured by SMC of low, intermediated, and high dose and ox-LDL coculture and ox-LDL alone. (b) MDA activity measured by SMC of low, intermediated, and high dose and ox-LDL coculture and ox-LDL single. The control group was treated with SMC alone; the ox-LDL group was treated with ox-LDL and SMC. The SOD activity of ox-LDL was lower than that of the other groups $(P<0.01)$, and different dose groups of SMC increased the decrease in the SOD value caused by oxLDL to varying degrees. MDA in the ox-LDL group was higher than the other groups $(P<0.01)$, and different dose groups of SMC reduced the increase in MDA values caused by ox-LDL to varying degrees. Data are presented as mean \pm standard deviation. ${ }^{*} P<0.05$; ${ }^{* *} P<0.01$. ox-LDL, oxidized low-density lipoprotein; low, low dose of SMC; intermediated, intermediated dose of SMC; high, high dose of SMC.

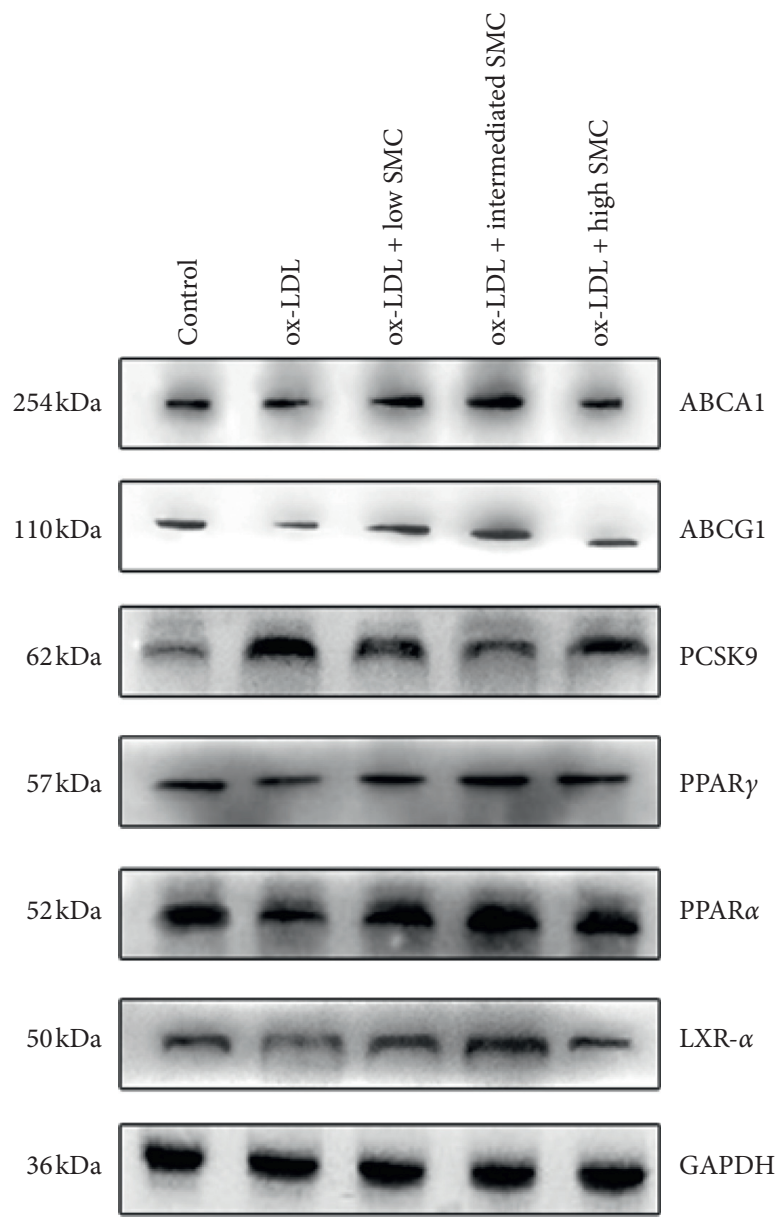

(a)

Figure 6: Continued. 


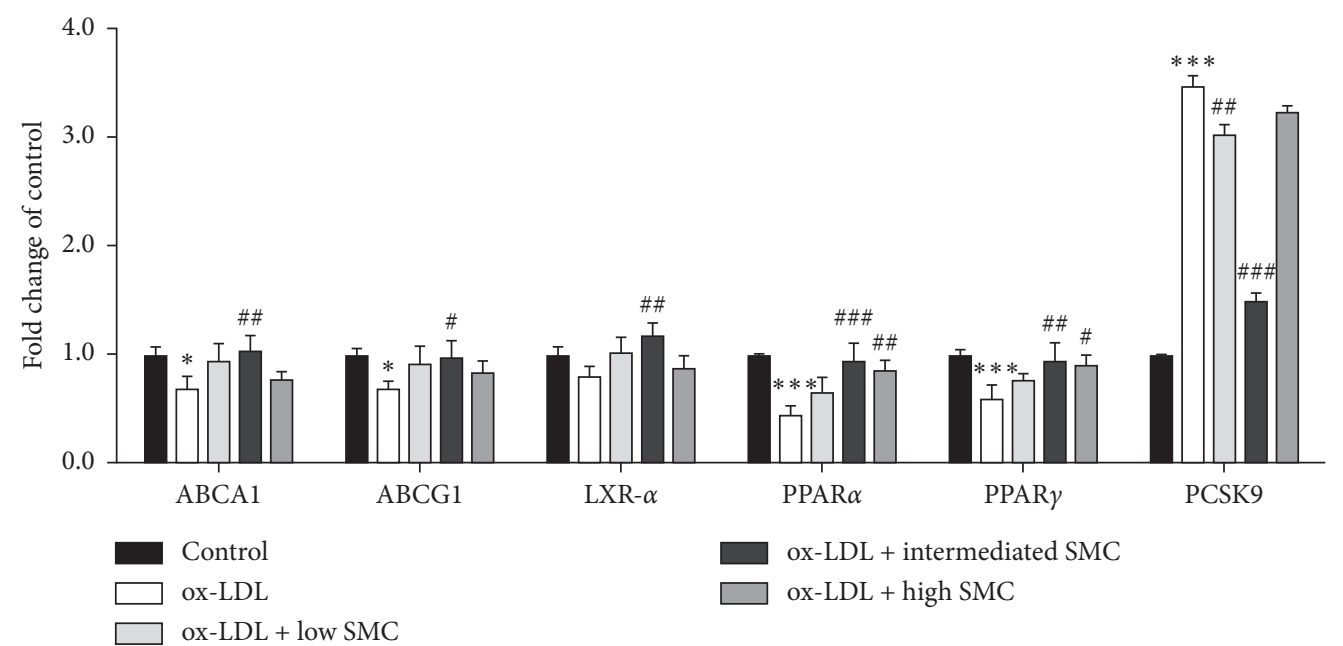

(b)

FIGURE 6: Western blot analysis of the expression of ABCA1, ABCG1, LXR $\alpha$, PPAR $\alpha$, PPAR $\gamma$, and PCSK9 in RAW264.7 cells. GAPDH was served as an internal control of the protein level. Determined expression of the protein was subsequently quantified by densitometric analysis with that of control being 1.00-fold. The expression of ABCA1, ABCG1, LXR $\alpha, \operatorname{PPAR} \alpha$, and PPAR $\gamma$ was decreased, induced by oxLDL, but the expression of PCSK9 was increased, induced by ox-LDL. After treatment with SMC, ABCA1, ABCG1, LXR $\alpha$, PPAR $\alpha$, and PPAR $\gamma$ expressions were upregulated and the expression of PCSK9 was downregulated. Results are representative of at least three independent experiments.

macrophage lipid accumulation cell model, demonstrating that SMC can reduce lipids in macrophages. However, our data showed that the intermediated dose of SMC had the best effect on reducing the lipid accumulation, lipid droplets, intracellular cholesterol, and total cholesterol content of RAW264.7 cells. This possibly results because the high dose had a certain cytotoxic effect, which reduced the efflux of lipids in cells.

The preparation has been used clinically for nearly 20 years and has good clinical anti-AS effect. According to the existing research literature, previous reports have shown that SMC treatment can effectively change the blood lipid composition by reducing serum TG, TC, and LDL, increasing HDL, and eventually delaying atherosclerotic plaque formation [23-26]. Moreover, studies have shown that SMC can prevent and treat atherosclerosis by regulating the inflammatory response mediated by the NF- $\kappa$ B signaling pathway [27]. However, this study is the first time to investigate the mechanism of action of the SMC from the perspective of in vitro cell research. Previous studies and conclusions are based on the results of clinical experiments, and the efficacy is usually judged by performing serum detection and measurement of atherosclerotic plaque size. Also, previous studies have shown that SMC has a good effect on atherosclerosis, but it has not specifically explored the molecular pathway mechanism that may have an effect. In this study, we explored a possible mechanism by which SMC reduces intracellular lipid accumulation by establishing ox-LDLinduced macrophage-derived foam cell models. In our study, treating cells with SMC can reduce intracellular lipid levels and promote cholesterol transport by affecting the expression of proteins related to cholesterol transport.
The reverse transport of cholesterol is mainly mediated by ABCA1 and ABCG1 [34, 35]. LXR $\alpha$ agonists upregulate the expression of $A B C A 1$ and ABCG1 and inhibit the differentiation of macrophages into foam cells by promoting cellular efflux [36-38]. LXR $\alpha$ expression was modulated by nuclear receptors, particularly PPARs, and many studies have determined that PPAR $\gamma$ agonists can induce LXR $\alpha$ expression in human and murine macrophages [39-41]. Based on our western blotting results, it can be speculated that SMC may reduce foam cell formation by increasing the expression of cholesterol efflux transporters. This may be related to the effective regulation of ABCA1-related pathways.

Our test results of SOD and MDA found that SMCs in different dose groups can increase the SOD value of cells treated with ox-LDL and decrease the MDA value. SOD and MDA are classic indicators of oxidative stress evaluation, and there are currently reports in the literature that oxidative stress can promote cellular senescence [42-44]. Studies show that oxidative stress accelerate telomere shortening or dysfunction and telomere shortening suppresses cancer by triggering senescence in normal cells [45-47]. Therefore, our results can deduce that SMC has a possible role in delaying cellular senescence.

In conclusion, as a traditional Chinese medicine preparation, SMC can be used for treating AS, which can block ox-LDL-induced damage of RAW264.7 cells, improve cell viability, and reduce lipid accumulation and senescence. This may be related to the effective regulation of ABCA1related pathways, PPAR $\alpha$-LXR $\alpha$-ABCA1, PPAR $\gamma$-LXR $\alpha$ ABCA1, and LXR $\alpha$-ABCA1, and the expression of proteins such as ABCG1, LXR $\alpha$, and PCSK9. Future research will continue to study the effects of SMC on the expression of RCT-related proteins. 


\section{Abbreviations}

SMC: $\quad$ Shenqi Maixintong capsule

AS: $\quad$ Atherosclerosis

ox- Oxidized low-density lipoprotein

LDL:

LDL-C: Low-density lipoprotein cholesterol

ABCA1: ATP-binding cassette transporter 1

ABCG1: ATP-binding cassette subfamily G member 1

LXR $\alpha$ : Liver X receptor alpha

PPAR $\alpha$ : Peroxisome proliferation-activated receptor alpha

PPAR $\gamma$ : Peroxisome proliferator-activated receptor $\gamma$

PCSK9: Proprotein convertase subtilisin/kexin type 9

HPLC: High-performance liquid chromatography

SOD: $\quad$ Superoxide dismutase

MDA: Malondialdehyde

HDL: High-density lipoprotein

RCT: Reverses cholesterol transport

HDL-C: High-density lipoprotein cholesterol

LXR: Liver $\mathrm{X}$ receptors

IOD: Integrated optical density

LDL-C: Low-density lipoprotein cholesterol

SD: $\quad$ Sprague Dawley

SPF: $\quad$ Specific pathogen-free

SRs: $\quad$ Scavenger receptors.

\section{Data Availability}

All data analyzed during this study are included in the manuscript. The raw data used and analyzed during the current study are available from the corresponding author upon reasonable request.

\section{Ethical Approval}

All procedures described in this study were performed in accordance with the guidelines of the Institutional Animal Ethical Committee (Jinan University of Experimental Animal Center).

\section{Conflicts of Interest}

The authors have no conflicts of interest regarding the publication of this paper.

\section{Authors' Contributions}

Qianqian Ma was responsible for sourcing of research articles, designing and performing the experiments, writing original draft, reviewing, and editing. Min Liang carried out compiling and writing of original draft, reviewing, and editing. Tiange Cai did reviewing and cross verification of research articles, writing review, and editing. Yingjia Yu, Xinjian Liang, Qingzhuang Chen, and Manling Du were responsible for experiment and data arrangement. Yong Ouyang and Chaojun Chen made supervision and editing of the final draft. Yu Cai carried out supervision and final editing of review. Q. Ma, M. Liang, and T. Cai have contributed equally to this manuscript.

\section{Acknowledgments}

This study was financially supported by the Traditional Chinese Medicine Bureau of Guangdong Province (20181215).

\section{References}

[1] G. S. Getz and C. A. Reardon, "The mutual interplay of lipid metabolism and the cells of the immune system in relation to atherosclerosis," Clinical Lipidology, vol. 9, no. 6, pp. 657-671, 2014.

[2] C. Wu, H. Luan, X. Zhanga et al., "Chlorogenic acid protects against atherosclerosis in ApoE-/- mice and promotes cholesterol efflux from RAW264.7 macrophages," PLoS One, vol. 9, no. 9, Article ID e95452, 2014.

[3] M. Wang, D. Wang, Y. Zhang, X. Wang, Y. Liu, and M. Xia, "Adiponectin increases macrophages cholesterol efflux and suppresses foam cell formation in patients with type 2 diabetes mellitus," Atherosclerosis, vol. 229, no. 1, pp. 62-70, 2013.

[4] F. B. Jan, K. Folkert, and K. G. Albert, "Cholesterol transport revisited: a new turbo mechanism to drive cholesterol excretion," Trends in Endocrinology and Metabolism, vol. 29, no. 2, pp. 123-133, 2018.

[5] X.-H. Yu, Y.-C. Fu, D.-W. Zhang, K. Yin, and C.-K. Tang, "Foam cells in atherosclerosis," Clinica Chimica Acta, vol. 424, pp. 245-252, 2013.

[6] X.-W. He, D. Yu, W.-L. Li et al., "Anti-atherosclerotic potential of baicalin mediated by promoting cholesterol efflux from macrophages via the PPAR $\gamma$-LXR $\alpha$-ABCA1/ABCG1 pathway," Biomedicine \& Pharmacotherapy, vol. 83, pp. 257-264, 2016.

[7] S. Ikhlef, H. Berrougui, O. Kamtchueng Simo, and A. Khalil, "Paraoxonase 1-treated oxLDL promotes cholesterol efflux from macrophages by stimulating the PPAR $\gamma$-LXR $\alpha$-ABCA1 pathway," Febs Letters, vol. 590, no. 11, pp. 1614-1629, 2016.

[8] S. Kersten, "Integrated physiology and systems biology of PPAR $\alpha$," Molecular Metabolism, vol. 3, no. 4, pp. 354-371, 2014.

[9] Y. Xu, F. Lai, Y. Xu et al., "Mycophenolic acid induces ATPbinding cassette transporter A1 (ABCA1) expression through the PPAR $\gamma$-LXR $\alpha$-ABCA1 pathway," Biochemical and Biophysical Research Communications, vol. 414, no. 4, pp. 779782, 2011.

[10] A. Chawla, W. A. Boisvert, C.-H. Lee et al., "A PPAR $\gamma$-LXRABCA1 pathway in macrophages is involved in cholesterol efflux and atherogenesis," Molecular Cell, vol. 7, no. 1, pp. 161-171, 2001.

[11] A. F. Edward, E. F. Jonathan, and S. Raanan, "Atheroprotective effects of HDL: beyond reverse cholesterol transport," Current Drug Targets, vol. 9, no. 3, pp. 196-203, 2008.

[12] L. Yvan-Charvet, N. Wang, and A. R. Tall, "Role of HDL, ABCA1, and ABCG1 transporters in cholesterol efflux and immune responses," Arteriosclerosis, Thrombosis, and Vascular Biology, vol. 30, no. 2, pp. 139-143, 2010.

[13] X. Wang, H. L. Collins, M. Ranalletta et al., "Macrophage ABCA1 and ABCG1, but not SR-BI, promote macrophage reverse cholesterol transport in vivo," Journal of Clinical Investigation, vol. 117, no. 8, pp. 2216-2224, 2007.

[14] M. A. Kennedy, G. C. Barrera, K. Nakamura et al., "ABCG1 has a critical role in mediating cholesterol efflux to HDL and preventing cellular lipid accumulation," Cell Metabolism, vol. 1, no. 2, pp. 121-131, 2005. 
[15] E. P. Demina, V. V. Miroshnikova, and A. L. Schwarzman, "Role of the ABC transporters A1 and G1, key reverse cholesterol transport proteins, in atherosclerosis," Molecular Biology, vol. 50, no. 2, pp. 193-199, 2016.

[16] K. Ohara, H. Wakabayashi, Y. Taniguchi, K. Shindo, H. Yajima, and A. Yoshida, "Quercetin-3-O-glucuronide induces ABCA1 expression by $\operatorname{LXR} \alpha$ activation in murine macrophages," Biochemical and Biophysical Research Communications, vol. 441, no. 4, pp. 929-934, 2013.

[17] P. Costet, Y. Luo, N. Wang, and A. R. Tall, "Sterol-dependent transactivation of the $\mathrm{ABC1}$ promoter by the liver $\mathrm{X}$ receptor/ retinoid X receptor," Journal of Biological Chemistry, vol. 275, no. 36, pp. 28240-28245, 2000.

[18] X. Ma, S.-F. Li, Z.-S. Qin et al., "Propofol up-regulates expression of ABCA1, ABCG1, and SR-B1 through the PPAR $\gamma /$ LXR $\alpha$ signaling pathway in THP-1 macrophage-derived foam cells," Cardiovascular Pathology, vol. 24, no. 4, pp. 230-235, 2015.

[19] A. Majdalawieh and H. S. Ro, "PPAR $\gamma 1$ and $L X R \alpha$ face a new regulator of macrophage cholesterol homeostasis and inflammatory responsiveness, AEBP1," Nuclear Receptor Signaling, vol. 16, no. 1, 2010.

[20] H. Oberkofler, E. Schraml, F. Krempler, and W. Patsch, "Potentiation of liver X receptor transcriptional activity by peroxisome-proliferator-activated receptor gamma co-activator 1alpha," Biochemical Journal, vol. 371, no. 1, pp. 89-96, 2003.

[21] F. Paciullo, F. Fallarino, V. Bianconi, M. R. Mannarino, A. Sahebkar, and M. Pirro, "PCSK9 at the crossroad of cholesterol metabolism and immune function during infections," Journal of Cellular Physiology, vol. 232, no. 9, pp. 2330-2338, 2017.

[22] M. P. Adorni, E. Cipollari, E. Favari et al., "Inhibitory effect of PCSK9 on Abca1 protein expression and cholesterol efflux in macrophages," Atherosclerosis, vol. 256, pp. 1-6, 2017.

[23] C. J. Chen, J. Y. Li, J. F. Hu, M. Du, and Y. Ouyang, "Effect of shenqimaixintong capsule on atherosclerosis in patients with carotid arteriosclerosis," Guangdong Medical Journal, vol. 37, no. 8, pp. 1228-1230, 2016.

[24] W. J. Huang and C. J. Chen, "Effect of shenqimaixintong capsule on stabilization of atherosclerotic plaque in cervical artery," Journal of Traditional Chinese Medicine, vol. 48, no. 9, p. 821, 2007.

[25] J. F. Hu, C. J. Chen, Z. H. Yu et al., "Effect of shenqimaixintong capsule on vascular endothelial function in patients with carotid arteriosclerosis," Chinese Journal of Integrated Traditional and Western Medicine, vol. 36, no. 8, pp. 10101012, 2016.

[26] K. R. Luo and C. J. Chen, "Effect of Shenqi Maixintong capsule on monocyte related cytokines in patients with carotid atherosclerosis," Shandong Journal of Traditional Chinese Medicine, vol. 38, no. 4, pp. 331-334+346, 2019.

[27] Y. M. Luo, C. J. Chen, and Y. J. Li, "Study on the mechanism of ShenQi MaiXinTong capsules fighting against atherosclerosis based on NF- $\kappa \mathrm{B}$," Western Journal of Traditional Chinese Medicine, vol. 31, no. 5, pp. 8-10, 2018.

[28] J. F. Hu, C. J. Chen, Z. H. Yu et al., "Influence of Shenqi maixitong capsule on atherosclerotic plaque stability in patients with carotid artery atherosclerosis," Journal of Liaoning University of Traditional Chinese Medicine, vol. 16, no. 11, pp. 60-62, 2014.

[29] C. J. Chen, J. Fu, and W. J. Huang, "Effect of shenqimaixintong capsule on dendritic cell antigen-presenting related endothelial damage factor," Guangdong Medical Journal, vol. 30, no. 6, pp. 888-890, 2009.

[30] S. Y. Xu, R. L. Bian, and X. Chen, Pharmacological Experimental Methodology, People's Health Publishing House, Beijing, China, 3rd edition, 2001.

[31] Y. Y. Yang, X. Y. Li, L. Y. Peng et al., "Tanshindiol C inhibits oxidized low-density lipoprotein induced macrophage foam cell formation via a peroxiredoxin 1 dependent pathway," Biochimica et Biophysica Acta-Molecular Basis of Disease, vol. 1864, no. 4, pp. 882-890, 2018.

[32] S. Gao and J. Liu, "Association between circulating oxidized low-density lipoprotein and atherosclerotic cardiovascular disease," Chronic Diseases and Translational Medicine, vol. 3, no. 2, pp. 89-94, 2017.

[33] D. Steinberg, "Low density lipoprotein oxidation and its pathobiological significance," Journal of Biological Chemistry, vol. 272, no. 34, pp. 20963-20966, 1997.

[34] X.-X. Liu, X.-W. Zhang, K. Wang et al., "Kuwanon G attenuates atherosclerosis by upregulation of $\operatorname{LXR} \alpha-\mathrm{ABCA} 1 /$ ABCG1 and inhibition of NF $\kappa$ B activity in macrophages," Toxicology and Applied Pharmacology, vol. 341, pp. 56-63, 2018.

[35] Y. He, L. Lin, J. Cao et al., "Up-regulated miR-93 contributes to coronary atherosclerosis pathogenesis through targeting ABCA1," International Journal of Clinical and Experimental Medicine, vol. 8, no. 1, pp. 674-681, 2015.

[36] Y.-W. Hu, Q. Wang, X. Ma et al., "TGF-\&beta;1 up-regulates expression of ABCA1, ABCG1 and SR-BI through liver $\mathrm{X}$ receptor \&alpha; signaling pathway in THP-1 macrophagederived foam cells," Journal of Atherosclerosis and Thrombosis, vol. 17, no. 5, pp. 493-502, 2010.

[37] R. Geyeregger, M. Zeyda, and T. M. Stulnig, "Liver x receptors in cardiovascular and metabolic disease," Cellular and Molecular Life Sciences, vol. 63, no. 5, pp. 524-539, 2006.

[38] K. Schwartz, R. M. Lawn, and D. P. Wade, "Abc1 gene expression and apoa-i-mediated cholesterol efflux are regulated by lxr," Biochemical and Biophysical Research Communications, vol. 274, no. 3, pp. 794-802, 2000.

[39] R. K. Tangirala, E. D. Bischoff, S. B. Joseph et al., "Identification of macrophage liver $\mathrm{x}$ receptors as inhibitors of atherosclerosis," Proceedings of the National Academy of Sciences, vol. 99, no. 18, pp. 11896-11901, 2002.

[40] A. Venkateswaran, B. A. Laffitte, S. B Joseph et al., "Control of cellular cholesterol efflux by the nuclear oxysterol receptor lxr alpha," PNAS, vol. 97, no. 2, pp. 12097-12102, 2000.

[41] L. Nagy, P. Tontonoz, J. G. A. Alvarez, H. Chen, and R. M. Evans, "Oxidized LDL regulates macrophage gene expression through ligand activation of PPAR $\gamma$," Cell, vol. 93, no. 2, pp. 229-240, 1998.

[42] E. Fouquerel, R. P. Barnes, S. Uttam, S. C. Watkins, M. P. Bruchez, and P. L. Opresko, "Targeted and persistent 8oxoguanine base damage at telomeres promotes telomere loss and crisis," Molecular Cell, vol. 75, no. 1, pp. 117.e6-130.e6, 2019.

[43] W. M. Chen, J. C. Chiang, Y. C. Lin et al., "“'Lysophosphatidic acid receptor LPA3 prevents oxidative stress and cellular senescence in Hutchinson-Gilford progeria syndrome," Aging Cell, vol. 19, Article ID e13064, 2020.

[44] M. Baeeri, H. Bahadar, M. Rahimifard et al., " $\alpha$-Lipoic acid prevents senescence, cell cycle arrest, and inflammatory cues in fibroblasts by inhibiting oxidative stress," Pharmacological Research, vol. 141, pp. 214-223, 2019. 
[45] J. Maciejowski and T. de Lange, "Telomeres in cancer: tumour suppression and genome instability," Nature Reviews Molecular Cell Biology, vol. 18, no. 3, pp. 175-186, 2017.

[46] R. P. Barnes, E. Fouquerel, and P. L. Opresko, "The impact of oxidative DNA damage and stress on telomere homeostasis," Mechanisms of Ageing and Development, vol. 177, pp. 37-45, 2019.

[47] W. Ahmed and J. Lingner, "Impact of oxidative stress on telomere biology," Differentiation, vol. 99, pp. 21-27, 2018. 